\title{
Multinational Observational Study to Understand Insulin Treatment Intensification: Japanese Subgroup Analysis of the MOSAIc Study
}

\author{
Ikuro Matsuba - Takehiro Kawata - Miho Ajima - Shinichi Umezawa • \\ Mizuki Kaneshiro • Taro Asakura • Hideo Machimura • \\ Tasuku Sawa $\cdot$ Keiji Tanaka · Hiroshi Takeda · Takeshi Imaoka • \\ Akira Kanamori
}

Received: September 2, 2021 / Accepted: December 15, 2021 / Published online: January 6, 2022

(C) The Author(s) 2022

\section{ABSTRACT}

Introduction: MOSAIc was a multinational, non-interventional, prospective, observational cohort study designed to provide an understanding of the specific challenges associated with intensification of initial insulin therapy in patients with type 2 diabetes mellitus (T2DM). We present a sub-analysis of Japanese patients from MOSAIc, with data analyzed longitudinally over 2 years, to provide insight on how T2DM treatment is intensified.

Methods: Japanese patients with T2DM receiving any insulin therapy for at least 3 months were eligible for study inclusion. Baseline and clinical data were collected during an initial

I. Matsuba · T. Kawata · S. Umezawa .

M. Kaneshiro · T. Asakura · H. Machimura .

K. Tanaka $\cdot$ H. Takeda $\cdot$ A. Kanamori

The Study Group of the Diabetes Committee,

Kanagawa Physicians Association, Kanagawa, Japan

M. Ajima

Department of Diabetology, Kawasaki Saiwai Clinic, Kanagawa, Japan

T. Sawa

Sawa Internal Medicine and Diabetes Clinic, Kanagawa, Japan

T. Imaoka $(\bowtie)$

Japan Drug Development and Medical Affairs, Eli Lilly Japan K.K., 5-1-28 Isogami-dori, Chuo-ku,

Kobe, Hyogo 651-0086, Japan

e-mail: imaoka_takeshi@lilly.com baseline visit and during four subsequent prospective visit windows (within \pm 3 months) at $6,12,18$, and 24 months. Treatment intensification was defined as addition of new insulin, increase in insulin dosage (1-unit change or $10 \%$ compared with the previous visit), increase in insulin injection frequency, and/or addition of non-insulin antihyperglycemic agents.

Results: Of 116 Japanese patients who completed the study, $50.0 \%(n=58)$ received treatment intensification. Baseline characteristics of patients with treatment intensification included a longer duration of diabetes, higher incidence of baseline microvascular complications, and higher HbA1c compared to those without intensification. There was no significant difference in HbA1c change from baseline between the two groups at any post-baseline visit. Insulin intensification accounted for $61.2 \%$ of treatment changes, with non-insulin-related intensification accounting for $36.2 \%$ of treatment changes. An increase in insulin dose was the most frequent treatment change $(51.7 \%)$, followed by the addition of new insulin $(22.4 \%)$, and an increase in insulin injection frequency $(6.9 \%)$.

Conclusion: Real-world data from Japanese patients with T2DM who received treatment intensification showed that an increase in insulin dose and the addition of new insulin were the most frequent treatment intensification methods. HbA1c was maintained through 2 years of treatment. 
Trial Registration: NCT01400971, ClinicalTrials.gov.

Keywords: Diabetes mellitus; HbA1c; Insulin; Japan

\section{Key Summary Points}

Why carry out this study?

Insulin is one of the major pillars of treatment for type 2 diabetes mellitus (T2DM) and effective even for people who failed to achieve satisfactory blood glucose control with other antidiabetic drugs; however, treatment intensification is often required to manage blood glucose control long term.

There are no reports in Japan on how physicians intensify insulin treatment in clinical practice.

We report the results of the Japanese subgroup analysis from the MOSAIc study, which shows how physicians intensify insulin treatment in patients with T2DM in real-world settings.

\section{What was learned from the study?}

Real-world data from Japanese patients with T2DM who received treatment intensification showed that an increase in insulin dose and the addition of new insulin are the most frequent treatment intensification methods.

Satisfactory blood glucose levels were maintained through 2 years of treatment.

\section{INTRODUCTION}

The incidence and prevalence of type 2 diabetes mellitus (T2DM) continue to rapidly increase [1], particularly in Asian countries [2]. The diabetes epidemic in Asia is characterized by a relatively young age of onset, intensifying the need for effective management strategies to minimize the burden of diabetes-associated care on healthcare systems [2].

Maintenance of glycemic control, as measured by glycated hemoglobin A1c (HbA1c) level, is the primary goal of treatment for T2DM and is associated with improved health outcomes for patients [3]. As a result of the progressive nature of T2DM, many patients progress to the point at which basal insulin alone is insufficient, with such patients requiring additional therapeutic support [4]. In clinical practice, insulin switching and/or intensification (such as increasing the frequency of dosing, the addition of rapid-acting insulin, or a switch to premix insulin) is required in patients when glycemic targets become unattainable using their current treatment regimen [5-8].

Despite the efficacious nature of insulin therapy, patients frequently encounter challenges in maintaining target HbA1c goals, suggesting that there may be challenges in treatment progression from initial insulin therapy [9]. Existing research has described barriers to insulin initiation and adherence [10-12]; however, data are limited regarding the unique challenges associated with insulin intensification in clinical practice $[9,13]$. There are currently no reports in Japan on how physicians intensify insulin treatment for patients with T2DM in the real-world setting.

The MOSAIc study was a multinational, noninterventional, prospective, observational cohort study designed to provide an understanding of the specific challenges associated with progression of initial insulin therapy to mealtime and more advanced insulin therapy, from the perspective of both the patient and the physician [9]. We present a sub-analysis of Japanese patients from MOSAIc, with data analyzed at baseline and longitudinally over 2 years to provide insight on how T2DM treatment is intensified. The identification of factors that impact treatment progression may lead to the development of more targeted patient- and physician-centered strategies and interventions to improve glycemic control in patients with T2DM. Improved glycemic control will ultimately decrease the risk of diabetes-related 
complications, reduce the need and consequent costs associated with secondary intervention and chronic care, and improve overall management and quality of life for Japanese patients with T2DM.

\section{METHODS}

\section{Study Design}

A detailed description of the rationale and design of the MOSAIc study has been reported [9]. Briefly, MOSAIc was a multinational, noninterventional, prospective, observational cohort study conducted in 18 countries, including Japan. After study enrollment, patients were observed for 2 years with visits approximately $6,12,18$, and 24 months following the baseline visit, with all visits being part of the patients' routine care.

The study was conducted in accordance with the ethical principles that have their origin in the Declaration of Helsinki and that are consistent with good clinical practices and applicable laws. The MOSAIc study was registered under ClinicalTrials.gov (NCT01400971) and the study protocol was reviewed and approved by institutional ethics committees at each study center. All participants provided written informed consent.

\section{Study Population}

Inclusion criteria for participation in MOSAIc were age at least 18 years; diagnosis of T2DM; presentation to a study site as part of usual medical care; and use of any commercially available initial insulin therapy for at least 3 months with or without any combination of approved non-insulin oral antidiabetic (OADs) drugs. Exclusion criteria were participation in another medical research study that included an investigational drug/procedure; use of intensive basal-bolus therapy (basal insulin in addition to three prandial doses); or initiation of insulin treatment with three daily injections of mixed insulin.

\section{Data Collection}

Data collection occurred during an initial baseline visit and during four subsequent prospective visit windows (within \pm 3 months) at 6,12 , 18 , and 24 months. There were no additional treatments, visits, or laboratory collections required beyond those occurring within the course of normal care. Patients were able to discontinue study participation at any time. At baseline, retrospective data were collected for the period beginning 6 months prior to baseline. Baseline data relating to T2DM clinical history, including diagnosis, treatment and complications, and medication history, were collected from medical records. Most recently recorded laboratory values were assessed but limited to the period 6 months prior to the baseline visit.

\section{Treatment Progression}

Insulin progression was defined on the basis of each patient's insulin therapy regimen at the baseline visit. If a patient commenced the study on basal insulin, with or without any other non-insulin antidiabetic medication, progression was defined as the addition of prandial insulin, an increased frequency of insulin injections, change to an insulin mixture, addition of a glucagon-like peptide 1 (GLP-1) medication, or at least one OAD medication. Alternatively, if a patient began the study on an insulin regimen of basal insulin plus fewer than three prandial injections daily or was using insulin mixtures (both regimens may be used alone or with other non-insulin antidiabetic medications), progression was defined as an increase in frequency of insulin injections, change to a basal-bolus regimen, or addition of a GLP-1 medication or at least one OAD medication. Importantly, this definition allowed for the inclusion of patients with T2DM who may be using insulin pumps, an emerging treatment approach among such patients.

Treatment change was defined as the change of insulin type, insulin dosage (10\% or 1 -unit change from the previous visit), insulin frequency, and/or employing non-insulin 
antihyperglycemic agents. Treatment initiation or changes were solely at the discretion of the physician and the patient.

Descriptive summary analyses (mean, standard deviation [SD], and frequency) were reported for patient baseline characteristics, clinical variables, and laboratory values. Patients with or without treatment intensification were evaluated at visit 5 . Only patients with both baseline and visit 5 (month 24) data were included.

\section{RESULTS}

\section{Patient Demographics and Baseline Characteristics}

A total of 153 patients were enrolled in Japan. Overall, 116 patients completed the study; half $(n=58,50.0 \%)$ received treatment intensification during the 2-year study period while the other half $(n=58,50.0 \%)$ did not receive treatment intensification. The mean patient age was 62.3 years (SD 12.7 years), with a similar number of male and female patients in the two groups (patients with treatment intensification and patients without treatment intensification; Table 1).

Mean baseline HbA1c was significantly higher in patients with intensification $(p=0.047)$. Although not statistically significant, other variables which differed between the two patient groups at baseline were the incidence of microvascular complications (specifically retinopathy and nephropathy) and the use of sulfonylureas as OAD medications (higher in patients who received treatment intensification compared to those who did not receive treatment intensification).

\section{HbA1c}

At baseline, mean $\mathrm{HbA} 1 \mathrm{c}$ was $7.9 \%$ in patients with intensification and $7.4 \%$ in patients without treatment intensification. Throughout the 2-year observation period, HbA1c levels were less than $7.5 \%$ for patients without treatment intensification (Table 2). The change from baseline in $\mathrm{HbA1c}$ in patients with treatment intensification at visit 5 (month 24) was small $(0.1 \%)$, indicating that appropriate intensification of insulin was achieved during the 2-year study period.

\section{Treatment Intensification}

A summary of the number of insulin treatment intensifications or the addition of oral medication by visit is presented in Table 3 . At all visits, insulin treatment intensification (defined as a combination of injection frequency increase, addition of new insulin, or $10 \%$ or 1 unit of insulin dosage increase) was more common than the addition of oral medicines.

A summary of overall treatment intensification is presented in Table 4. Insulin intensification accounted for $61.2 \%$ of treatment changes while non-insulin-related intensification accounted for $36.2 \%$ of treatment changes. Among all patients, an increase in insulin dosage (10\% or 1 unit of insulin dosage change from the previous visit) was the most frequent treatment change, with over half of the patients $(51.7 \%)$ receiving an increase in insulin dosage. A total of $26(22.4 \%)$ patients received the addition of new insulin during the 2-year study. An increase in insulin injection frequency was experienced by $6.9 \%$ of patients.

\section{DISCUSSION}

Despite the fact that insulin intensification is recommended as part of the treatment for T2DM, there are limited studies that examine the trends of intensification, particularly among Asian countries. The pivotal MOSAIc study identified patient, physician, and healthcare environment-based factors associated with insulin progression for patients with T2DM in real-world practice [14]. Country-specific differences in patients' profiles and treatment patterns were identified, offering insight into the healthcare environment in different geographic settings $[14,15]$. We report the findings of a Japanese subgroup analysis from the MOSAIc study, the first report regarding insulin intensification in this population. 
Table 1 Demographic and baseline characteristics of patients with and without treatment intensification

\begin{tabular}{llll}
\hline Variables & $\begin{array}{l}\text { Patients with treatment } \\
\text { intensification }(\boldsymbol{N}=\mathbf{5 8})\end{array}$ & $\begin{array}{l}\text { Patients without treatment } \\
\text { intensification }(\boldsymbol{N}=\mathbf{5 8})\end{array}$ & $\begin{array}{l}\text { Total } \\
(\boldsymbol{N}=\mathbf{1 1 6})\end{array}$ \\
\hline Sex, female, $n(\%)$ & $21(36.2)$ & $22(37.9)$ & $43(37.1)$ \\
Age, years & $61.6(12.8)$ & $63.0(12.7)$ & $62.3(12.7)$ \\
Baseline BMI, $\mathrm{kg} / \mathrm{m}^{2}$ & $24.9(4.1)$ & $25.3(4.7)$ & $25.2(4.4)$ \\
Duration of & $14.4(8.2)$ & $13.0(8.1)$ & $13.7(8.2)$ \\
diabetes, years & & & \\
Baseline microvascular complications, $n(\%)$ & $13(22.4)$ & $31(26.7)$ \\
Retinopathy & $18(31.0)$ & $11(19.0)$ & $29(25.0)$ \\
Nephropathy & $18(31.0)$ & $11(19.0)$ & $18(15.5)$ \\
Neuropathy & $7(12.1)$ & $7.4(1.3)$ & $7.7(1.3)$ \\
HbAlc, \% & $7.9(1.3)$ & & $51(44.0)$ \\
Oral antidiabetic medications, $n(\%)$ & $28(48.3)$ & $65(56.0)$ \\
Metformin & $23(39.7)$ & $27(46.6)$ & $49(42.2)$ \\
Sulfonylurea & $38(65.5)$ & $23(39.7)$ & $30(25.9)$ \\
Dipeptidyl & $26(44.8)$ & & $25(21.6)$ \\
peptidase 4 & $17(29.3)$ & $13(22.4)$ & $12(20.7)$ \\
Thiazolidinedione & $13(22.4)$ & & \\
Others & & & \\
\hline
\end{tabular}

Insulin treatment intensification was defined as a combination of injection frequency increase, addition of new insulin, or $10 \%$ or 1 unit of insulin dosage increase

Unless indicated otherwise, data are presented as the mean $( \pm S D)$

$B M I$ body mass index, $H b A 1 c$ glycated hemoglobin Alc, $N$ total number of patients in specified treatment, $n$ number of patients in specified category, $S D$ standard deviation

As a result of the progressive nature of $\mathrm{T} 2 \mathrm{DM}$, an increasingly intensive treatment strategy is needed to achieve glycemic control over time, with likely insulin intensification. In our analysis of Japanese patients, baseline characteristics of patients with treatment intensification included a higher incidence of baseline microvascular complications, higher usage of sulfonylureas, and higher HbA1c. HbA1c values appear to be consistently higher in the treatment intensification group compared with the non-intensification group during the 2-year study period. A 12-week observational study in Japanese patients with T2DM suggested that changes to insulin regimen may improve glycemic control without negatively impacting quality of life [16].

According to the Diabetes Mellitus Treatment Guidelines published by the Japan Diabetes Society, the glycemic goal is generally set at $\mathrm{HbA} 1 \mathrm{c}<7.0 \%$ but should be determined individually depending on various factors such as the disease condition, age of the patient, cognitive function, activities of daily living, and risk of severe hypoglycemia [17]. According to the results of this study, an HbA1c level of 7.5\% may be the level at which Japanese diabetologists consider intensifying insulin treatment.

Japanese guidelines for the management of T2DM recommend an individualized stepwise 
Table 2 Summary of HbAlc in patients with and without treatment intensification by visit

\begin{tabular}{|c|c|c|c|c|}
\hline Visit & $n$ & $\begin{array}{l}\text { Patients with treatment intensification } \\
(N=58)\end{array}$ & $n$ & $\begin{array}{l}\text { Patients without treatment intensification } \\
(N=58)\end{array}$ \\
\hline Baseline & 54 & $7.91(1.3)$ & 57 & $7.41(1.3)$ \\
\hline \multicolumn{5}{|c|}{ Visit 2 (month 6) } \\
\hline Actual value & 57 & $7.74(1.2)$ & 57 & $7.12(1.0)$ \\
\hline $\begin{array}{l}\text { Change from } \\
\text { baseline }\end{array}$ & 54 & $-0.14(1.1)$ & 56 & $-0.26(1.0)$ \\
\hline \multicolumn{5}{|c|}{ Visit 3 (month 12) } \\
\hline Actual value & 56 & $7.75(1.3)$ & 57 & $7.20(0.9)$ \\
\hline $\begin{array}{l}\text { Change from } \\
\text { baseline }\end{array}$ & 53 & $-0.06(1.0)$ & 56 & $-0.22(0.9)$ \\
\hline \multicolumn{5}{|c|}{ Visit 4 (month 18 ) } \\
\hline Actual value & 56 & $7.74(1.1)$ & 57 & $7.26(0.8)$ \\
\hline $\begin{array}{l}\text { Change from } \\
\text { baseline }\end{array}$ & 53 & $-0.08(0.9)$ & 56 & $-0.13(1.0)$ \\
\hline \multicolumn{5}{|c|}{ Visit 5 (month 24) } \\
\hline Actual value & 56 & $7.94(1.3)$ & 58 & $7.41(1.0)$ \\
\hline $\begin{array}{l}\text { Change from } \\
\text { baseline }\end{array}$ & 53 & $0.06(0.9)$ & 57 & $-0.01(1.1)$ \\
\hline
\end{tabular}

Insulin treatment intensification was defined as a combination of injection frequency increase, addition of new insulin, or $10 \%$ or 1 unit of insulin dosage increase

Unless indicated otherwise, data are presented as the mean $( \pm \mathrm{SD})$

HbAlc glycated hemoglobin A1c, $N$ total number of patients in specified treatment, $n$ number of patients in specified category, $S D$ standard deviation

approach to intensifying treatment [17]. There are several types of treatment intensification including an increase in insulin dose or frequency, or the addition of oral medication. In Japanese patients from MOSAIc, an increase in insulin dosage (10\% or 1 unit of insulin dosage change from the previous visit) was the most frequent treatment alteration, with over half of patients $(51.7 \%)$ receiving an increase in insulin dose. In addition, $36.2 \%$ of patients added on oral medications or employed non-insulin-related intensification. Japanese healthcare providers and patients may feel that an increase in insulin dose and/or addition of oral medications may be less of a burden and/or a relatively safe option for continued management of T2DM. The recent availability of GLP-1 receptor agonists, to be considered as a treatment option after oral medications but before insulin, provides an additional option for earlier intervention regarding HbA1c control in Japanese patients with T2DM.

The strength of the MOSAIc study lies in its real-world observations, with data collected during the course of usual care, thereby reflecting characteristics and patterns of patients with T2DM and their treatment in realworld settings. However, there are several limitations. This study did not evaluate patients' diet or exercise regime, or other medical and 
Table 3 Summary of insulin treatment intensification and the addition of oral medication by visit

\begin{tabular}{lll}
\hline Visit & Type, $\boldsymbol{n}(\%)$ & All patients $^{\mathbf{a}}(\boldsymbol{N}=\mathbf{1 1 6})$ \\
\hline Visit 2 (month 6) & Add on of oral medicines & $13(11.2)$ \\
Visit 3 (month 12) & Insulin treatment intensification & $46(39.7)$ \\
& Add on of oral medicines & $15(12.9)$ \\
Visit 4 (month 18) & Insulin treatment intensification & $49(42.2)$ \\
& Add on of oral medicines & $6(5.2)$ \\
Visit 5 (month 24) & Insulin treatment intensification & $47(40.5)$ \\
& Add on of oral medicines & $16(13.8)$ \\
Overall & Insulin treatment intensification & $50(43.1)$ \\
& Add on of oral medicines & $42(36.2)$ \\
\hline
\end{tabular}

Insulin treatment intensification was defined as a combination of injection frequency increase, addition of new insulin, or $10 \%$ or 1 unit of insulin dosage increase

${ }^{a}$ Patients may have two or more types of treatment intensification

Table 4 Summary of overall treatment intensification

\begin{tabular}{lc}
\hline Treatment, $\boldsymbol{n}(\%)$ & All patients p $^{\mathbf{a}}(\boldsymbol{N}=\mathbf{1 1 6})$ \\
\hline Insulin-related intensification & $71(61.2)$ \\
Add on of oral medicines (non-insulin related intensification) & $42(36.2)$ \\
Increase the injection frequency & $8(6.9)$ \\
Add on of new insulin & $26(22.4)$ \\
$10 \%$ or 1 unit of insulin dosage increase & $60(51.7)$ \\
Any 2 types of intensification & $37(31.9)$ \\
Any 3 types of intensification & $8(6.9)$ \\
All types of intensification & 0
\end{tabular}

Insulin treatment intensification was defined as a combination of injection frequency increase, addition of new insulin, or $10 \%$ or 1 unit of insulin dosage increase

${ }^{a}$ Patients may have two or more types of treatment intensification

lifestyle factors that may affect T2DM management. The rationale behind treatment decisions and the specific details of treatment intensification were not evaluated, nor were providerspecific barriers to insulin intensification. In addition, if patients consulted multiple healthcare providers, the full extent of their comorbidities and treatment may not be captured. 


\section{CONCLUSION}

In Japanese patients with T2DM participating in the MOSAIc study, an increase in insulin dose was the most frequent treatment intensification, with over half of the patients receiving an increase in insulin dosage. This sub-analysis showed that patients receiving treatment intensification maintained HbA1c levels through 2 years of treatment. This is the first real-world data concerning insulin intensification practices in Japanese patients with T2DM.

\section{ACKNOWLEDGEMENTS}

Funding. This study was funded by Eli Lilly and Company. The rapid service fee was funded by Eli Lilly Japan K.K.

Medical Writing Assistance. The authors thank Elizabeth Flate, PhD, and Lisa Cossens, $\mathrm{PhD}$, of Syneos Health for providing writing assistance and Antonia Baldo for editorial assistance, funded by Eli Lilly Japan K.K.

Authorship. All named authors meet the International Committee of Medical Journal Editors (ICMJE) criteria for authorship for this article, take responsibility for the integrity of the work as a whole, and have given their approval for this version to be published.

Author Contributions. Ikuro Matsuba, Takehiro Kawata, Miho Ajima, Shinichi Umezawa, Mizuki Kaneshiro, Taro Asakura, Hideo Machimura, Tasuku Sawa, Keiji Tanaka, Hiroshi Takeda and Akira Kanamori were involved in acquisition of the data, interpretation of the data, and critical revision of the work for important intellectual content. Takeshi Imaoka was involved in interpretation of the work and critical revision of the work for important intellectual content. All authors were involved in critical review of the manuscript.

Disclosures. Authors declare conflicts of interests with the following companies: Ikuro Matsuba with MSD K.K., AstraZeneca K.K.,
Kissei Pharmaceutical Co., Ltd., Sanofi K.K., Novo Nordisk Pharma Ltd., Pfizer Japan Inc., Sanwa Kagaku Kenkyusho Co., Ltd., Kyowa Kirin Co., Ltd., Sumitomo Dainippon Pharma Co., Ltd., Taisho Pharmaceutical Co., Ltd., Ono Pharmaceutical Co., Ltd., Mochida Pharmaceutical Co., Ltd., Eli Lilly Japan K.K., Nippon Boehringer Ingelheim Co., Ltd., Takeda Pharmaceutical Company Limited, Mitsubishi Tanabe Pharma Corporation; Takehiro Kawata with Ono Pharmaceutical Co., Ltd., Kyowa Kirin Co., Ltd., AstraZeneca K.K., Kissei Pharmaceutical Co., Ltd., Astellas Pharma Inc., Mitsubishi Tanabe Pharma Corporation; Miho Ajima with Taisho Pharmaceutical Co., Ltd., Ono Pharmaceutical Co., Ltd., Lifemedicom Co., Ltd., Taisho Pharma Co., Ltd., Sanwa Kagaku Kenkyusho Co., Ltd., Kowa Company, Ltd., Daiichi Sankyo Company, Ltd., Kissei Pharmaceutical Co., Ltd., Novo Nordisk Pharma Ltd., Eli Lilly Japan K.K.; Shinichi Umezawa with Kyowa Kirin Co., Ltd., Bayer Yakuhin, Ltd, Sanwa Kagaku Kenkyusho Co., Ltd., Mitsubishi Tanabe Pharma Corporation, Edwards Lifesciences Corporation, Astellas Pharma Inc., Ono Pharmaceutical Co., Ltd., Takeda Pharmaceutical Company Limited, Nippon Boehringer Ingelheim Co., Ltd., Novartis Pharma K.K., Sumitomo Dainippon Pharma Co., Ltd.; Mizuki Kaneshiro with Novartis Pharma K.K., Kyowa Kirin Co., Ltd., Sumitomo Dainippon Pharma Co., Ltd., Ono Pharmaceutical Co., Ltd., Nippon Boehringer Ingelheim Co., Ltd., Kowa Company, Ltd.; Taro Asakura with Novo Nordisk Pharma Ltd., Kyowa Kirin Co., Ltd., Sumitomo Dainippon Pharma Co., Ltd., Nippon Boehringer Ingelheim Co., Ltd., Kowa Company, Ltd., Taisho Pharmaceutical Co., Ltd; Hideo Machimura with MSD K.K., Astellas Pharma Inc, AstraZeneca K.K., Sanofi K.K., Novo Nordisk Pharma Ltd., Eli Lilly Japan K.K., Takeda Pharmaceutical Company Limited, Kowa Company, Ltd.; Tasuku Sawa with Ono Pharmaceutical Co., Ltd., Kyowa Kirin Co., Ltd., MSD K.K., Novartis Pharma K.K., Takeda Pharmaceutical Company Limited, Kowa Company, Ltd., Novo Nordisk Pharma Ltd., AstraZeneca K.K., Mitsubishi Tanabe Pharma Corporation, Kissei Pharmaceutical Co., Ltd., Sanofi K.K.; Keiji Tanaka with Takeda Pharmaceutical 
Company Limited, Daiichi Sankyo Company, Ltd., Sanwa Kagaku Kenkyusho Co., Ltd., Kyowa Kirin Co., Ltd., Nippon Boehringer Ingelheim Co., Ltd., Ono Pharmaceutical Co., Ltd., Sumitomo Dainippon Pharma Co., Ltd., Astellas Pharma Inc, Mitsubishi Tanabe Pharma Corporation, Taisho Pharmaceutical Co., Ltd., Kowa Company, Ltd., Novo Nordisk Pharma Ltd.; Hiroshi Takeda with AstraZeneca K.K., Novo Nordisk Pharma Ltd., Sanwa Kagaku Kenkyusho Co., Ltd., Kyowa Kirin Co., Ltd., Sumitomo Dainippon Pharma Co., Ltd., Taisho Pharmaceutical Co., Ltd., Eli Lilly Japan K.K., Mitsubishi Tanabe Pharma Corporation, Daiichi Sankyo Company, Ltd., Kowa Company, Ltd.; Takeshi Imaoka is a full-time employee at Eli Lilly Japan K.K. and a minor stockholder at Eli Lilly and Company.; Akira Kanamori has no conflicts of interest to declare.

Compliance with Ethics Guidelines. The study was conducted in accordance with the ethical principles of the Declaration of Helsinki and the International Council for Harmonisation Guideline for Good Clinical Practice. The MOSAIc study protocol was reviewed and approved by institutional ethics committees at each study center. All participants provided written informed consent.

Prior Presentation. Parts of this study were presented in abstract form at the 62nd Annual Meeting of the Japan Diabetes Society, Sendai, Japan, 23-25 May 2019.

Data Availability. Eli Lilly and Company provides access to all individual participant data collected during the trial, after anonymization, with the exception of pharmacokinetic or genetic data. Data are available to request 6 months after the indication studied has been approved in the USA and EU and after primary publication acceptance, whichever is later. No expiration date of data requests is currently set once data are made available. Access is provided after a proposal has been approved by an independent review committee identified for this purpose and after receipt of a signed data sharing agreement. Data and documents, including the study protocol, statistical analysis plan, clinical study report, and blank or annotated case report forms, will be provided in a secure data sharing environment. For details on submitting a request, see the instructions provided at www.vivli.org.

Open Access. This article is licensed under a Creative Commons Attribution-NonCommercial 4.0 International License, which permits any non-commercial use, sharing, adaptation, distribution and reproduction in any medium or format, as long as you give appropriate credit to the original author(s) and the source, provide a link to the Creative Commons licence, and indicate if changes were made. The images or other third party material in this article are included in the article's Creative Commons licence, unless indicated otherwise in a credit line to the material. If material is not included in the article's Creative Commons licence and your intended use is not permitted by statutory regulation or exceeds the permitted use, you will need to obtain permission directly from the copyright holder. To view a copy of this licence, visit http://creativecommons.org/licenses/by$\mathrm{nc} / 4.0 /$.

\section{REFERENCES}

1. Wild S, Roglic G, Green A, Sicree R, King H. Global prevalence of diabetes: estimates for the year 2000 and projections for 2030. Diabetes Care. 2004;27: 1047-53.

2. Chan JC, Malik V, Jia W, et al. Diabetes in Asia: epidemiology, risk factors, and pathophysiology. JAMA. 2009;301:2129-40.

3. American Diabetes Association. Standards of medical care in diabetes-2019 abridged for primary care providers. Clin Diabetes. 2019;37:11-34.

4. Meece J. Basal insulin intensification in patients with type 2 diabetes: a review. Diabetes Ther. 2018;9:877-90.

5. Nathan DM, Buse JB, Davidson MB, et al. Medical management of hyperglycemia in type 2 diabetes: a consensus algorithm for the initiation and adjustment of therapy: a consensus statement of the American Diabetes Association and the European 
Association for the Study of Diabetes. Diabetes Care. 2009;32:193-203.

6. Downie M, Kilov G, Wong J. Initiation and intensification strategies in type 2 diabetes management: a comparison of basal plus and premix regimens. Diabetes Ther. 2016;7:641-57.

7. Meneghini LF. Intensifying insulin therapy: what options are available to patients with type 2 diabetes? Am J Med. 2013;126:S28-37.

8. Mosenzon O, Raz I. Intensification of insulin therapy for type 2 diabetic patients in primary care: basal-bolus regimen versus premix insulin analogs. Diabetes Care. 2013;36(Suppl 2):S212-8.

9. Polinski JM, Curtis BH, Seeger JD, Choudhry NK, Zagar A, Shrank WH. Rationale and design of the multinational observational study assessing insulin use: the MOSAIc study. BMC Endocr Disord. 2012;12:20.

10. Peyrot M, Rubin RR, Lauritzen T, et al. Resistance to insulin therapy among patients and providers: results of the cross-national Diabetes Attitudes, Wishes, and Needs (DAWN) study. Diabetes Care. 2005;28:2673-9.

11. Polonsky WH, Arsenault J, Fisher L, et al. Initiating insulin: how to help people with type 2 diabetes start and continue insulin successfully. Int J Clin Pract. 2017;71:e12973.

12. Stuckey H, Fisher L, Polonsky WH, et al. Key factors for overcoming psychological insulin resistance: an examination of patient perspectives through content analysis. BMJ Open Diabetes Res Care. 2019;7: e000723.

13. Kunt T, Snoek FJ. Barriers to insulin initiation and intensification and how to overcome them. Int J Clin Pract Suppl. 2009;63:6-10.

14. Polinski JM, Kim SC, Jiang D, et al. Geographic patterns in patient demographics and insulin use in 18 countries, a global perspective from the multinational observational study assessing insulin use: understanding the challenges associated with progression of therapy (MOSAIC). BMC Endocr Disord. 2015;15:46.

15. Matsuba I, Sawa T, Kawata T, et al. Cross-national variation in glycemic control and diabetes-related distress among East Asian patients using insulin: results from the MOSAIc study. Diabetes Ther. 2016;7:349-60.

16. Ishii $H$, Terauchi $Y$, Jinnouchi $H$, Taketsuna $M$, Takeuchi M, Imaoka T. Effects of insulin changes on quality of life and glycemic control in Japanese patients with type 2 diabetes mellitus: the insulinchanging study intending to gain patients' insights into insulin treatment with patient-reported health outcomes in actual clinical treatments (INSIGHTs) study. J Diabetes Investig. 2013;4:560-70.

17. Araki E, Goto A, Kondo T, et al. Japanese clinical practice guideline for diabetes 2019. J Diabetes Investig. 2020;11:1020-76. https://doi.org/10. 1007/s13340-020-00439-5. 